\title{
Drug resistance and Cancer stem cells
}

\author{
Yuan $\mathrm{Li}^{1,2}$, Zhenning Wang ${ }^{2}$, Jaffer A. Ajani ${ }^{1 *}$ and Shumei Song ${ }^{1 *}$
}

\begin{abstract}
Therapy resistance is a major problem when treating cancer patients as cancer cells develop mechanisms that counteract the effect of therapeutic compounds, leading to fit and more aggressive clones that contribute to poor prognosis. Therapy resistance can be both intrinsic and/or acquired. These are multifactorial events, and some are related to factors including adaptations in cancer stem cells (CSCs), epithelial-mesenchymal transition (EMT), deregulation of key signaling pathways, drug efflux through ABC transporters, acquired mutations, evading apoptosis, and activation of DNA damage response among others. Among these factors, CSCs represent the major source of therapy resistance. CSCs are a subset of tumor cells that are capable of self-renewal and multilineage progenitor expansion that are known to be intrinsically resistant to anticancer treatments. Multiple clones of CSCs pre-exist, and some can adopt and expand easily to changes in the tumor microenvironment (TME) and/or in response to radio- and chemotherapy. A combination of both intrinsic and extrinsic factors contributes to CSCmediated therapy resistance. In this review, we will focus on CSCs and therapy resistance as well as suggest strategies to eliminate CSCs and, therefore, overcome resistance.
\end{abstract}

Keywords: Drug resistance, Cancer stem cells, EMT and TME

\section{Introduction}

Tumors harbor heterogeneous clones of cancer stem cells (CSCs) representing the fundamental properties for survival [1]. In recent years, addressing the origin of intratumor heterogeneity (ITH) has become one of the core challenges for overcoming therapy resistance. The clonal evolution model considers tumors to be the result of random evolution, while the CSC model considers tumor heterogeneity due to the presence of CSCs $[2,3]$. CSCs are defined as "cells that have the ability to selfrenew and also create a progeny". Therefore, the bulk of cancer mass is formed by the differentiated and expanded progeny with high proliferation potential while harboring small populations of various CSCs. At present, cure of tumor remains a major challenge for oncologists due to advanced stage of most cancers and the presence of resistant CSC clones $[4,5]$.

\footnotetext{
* Correspondence: jajani@mdanderson.org; ssong@mdanderson.org 'Department of Gastrointestinal Medical Oncology, The University of Texas MD Anderson Cancer Center, 1515 Holcombe Boulevard, Houston, TX 77030-4009, USA

Full list of author information is available at the end of the article
}

It has been proposed that targeting CSC subpopulations can result in tumor elimination thus no likelihood of tumor relapse [6]. CSCs evade conventional therapies by remaining dormant, have increased DNA repair capacity, turn off apoptotic pathways, manage reactive oxygen species (ROS) and reactive nitrogen species (RNS) in a highly competent manner, and by manipulating TME (tumor microenvironments) to their advantage [7].

\section{Potential CSCs markers and therapy resistance}

In essence, CSCs are defined by their intrinsic ability to propagate the tumor, thereby explaining the alternative names "tumor initiating cells" or "tumorigenic cells" [8]. CSCs can be identified by various surface markers. To date, several CSC markers in different tumor types have been proposed and validated through cell lines, patient samples, and xenotransplantation of CSCs in animal models. Followings are the CSC biomarkers of interest:

CD133, also known as prominin-1, a glycoprotein with five transmembrane domains, was identified from mouse neuro-epithelial stem cells and human hematopoietic

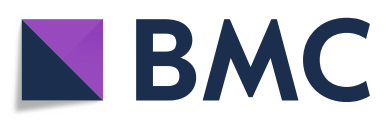

(C) The Author(s). 2021 Open Access This article is licensed under a Creative Commons Attribution 4.0 International License, which permits use, sharing, adaptation, distribution and reproduction in any medium or format, as long as you give appropriate credit to the original author(s) and the source, provide a link to the Creative Commons licence, and indicate if changes were made. The images or other third party material in this article are included in the article's Creative Commons licence, unless indicated otherwise in a credit line to the material. If material is not included in the article's Creative Commons licence and your intended use is not permitted by statutory regulation or exceeds the permitted use, you will need to obtain permission directly from the copyright holder. To view a copy of this licence, visit http://creativecommons.org/licenses/by/4.0/ The Creative Commons Public Domain Dedication waiver (http://creativecommons.org/publicdomain/zero/1.0/) applies to the data made available in this article, unless otherwise stated in a credit line to the data. 
stem cells. The expression of CD133 is not restricted to normal stem cell, but also found in many tumor types. Dirks et al. discovered that CD133 expressed in brain tumors and used CD133 as a CSC marker to identify brain CSCs [9]. CD133 was also identified in various other tumors including breast, stomach, colon, prostate, liver, pancreatic, ovarian, lung cancer, and head and neck squamous cell cancers [10-14]. CD133-expressing CSCs exhibited self-renewal potential and over-expression of CD133 has been associated with poor prognosis and reduced overall survival in gastric adenocarcinoma and several other tumor types [15].

High expression of CD133 is associated with drug resistance. The presence of CD133-positive CSCs in lung cancer increases the expression of the $\mathrm{ABC}$ transporter ABCG2, resulting in lung cancer resistance to first-line drugs such as platinum and paclitaxel. Studies have shown that low-dose platinum therapy can cause DNA damage rather than cell death, which can induce ABCG2 upregulation and further increase the number of CD133-positive cells. Specific ABCG2 inhibitor Pantoprazole or $A B C$ transporter inhibitor Verapamil can reduce tumor resistance to platinum [16]. It has been reported that CD133 mediates cisplatin resistance that can overcome by inhibition of CD133 [17].

$C D 44$, is a transmembrane receptor for hyaluronic acid (HA) and many other extra-cellular matrix (ECM) components and a coreceptor for growth factors and cytokines. It is reported that CD44 is associated with increased potential for tumor initiation and progression [18]. CD44 is an important cell surface molecule that can sense, integrate, and transduce cellular microenvironment signals to membrane-associated cytoskeleton proteins or to nuclei, thereby regulating the expression of various genes that control cell behaviors. Increasing evidence suggests that $\mathrm{CD} 44$, especially the $\mathrm{CD} 44 \mathrm{v}$ subtype, is a CSC biomarker and a key regulator of cancer stemness, metastases, and response to therapy [19].

$A L D H 1$, is a member of the aldehyde dehydrogenase (ALDH) superfamily of enzymes, which comprises 19 human isozymes. ALDH1 is known to participate in many important physiological biosynthetic pathways, and certain ALDH1 activities have been shown to be crucial in the detoxification of specific endogenous and exogenous aldehyde [20]. Many studies indicate that ALDH1 is involved in therapy resistance. ALDH1 controls the oxidation of aldehydes to corresponding acids, and ALDH-mediated detoxification of toxic aldehyde intermediates produced in cancer cells treated with certain therapeutic agents has been proposed to confer therapy resistance to ALDH1+ tumor cells [21]. In esophageal cancer, ALDH $1^{\text {high }}$ is associated with reduced response in chemoradiation and chemotherapy [22]. Meng's study indicates that ALDH1 expression is also correlated to the platinum resistance in ovarian cancer by regulating cell cycle checkpoints and the DNA repair pathway [23]. Using ALDH1 sorting, Nguyen et al. found that ALDH1+ CSCs had higher tumorigenicity in mice and were more resistant to therapy than ALDH1- gastric cancer cells [24]. Study from our laboratory also demonstrated that ALDH1 expression levels predict response or resistance to preoperative chemoradiation in resectable esophageal cancer patients and we found that sorted ALDH1+ cells were more resistant and had an aggressive phenotype than ALDH1- cells [22]. Targeted inhibition of ALDH1 could prevent recurrence of the tumor driven by ALDH1+ CSCs [25]. Therefore, as a detoxifying enzyme, ALDH1 is highly expressed in CSCs to alleviate the toxic effects of ROS and to control the cell cycle so that the cells have enough time for DNA repair, which enables CSCs to resist therapy.

$C D 166$, also named as activated leukocyte cell adhesion molecule (ALCAM), is a glycoprotein belonging to the immunoglobulin superfamily of adhesion molecules [26]. Unlike other adhesion molecules such as Ecadherin which are usually down-regulated during malignant transformation, CD166 often shows increased expression in certain cancers [27]. Overexpression of CD166 in papillary thyroid carcinoma (PTC) was independently associated with a shorter progression-free survival, higher nodal and tumor stages suggesting that CD166 may be a potential therapeutic target to treat PTC [28]. CD166 has been implicated as CSCs marker in many cancer types such as colon, stomach and head/ neck $[24,29]$. Recent study from Satar NA et al. demonstrated that CD166/EpCAM/CD44 triple positive clones mediated therapy resistance and putative CSC characteristics in human non-small cell lung cancer cells [30].

CD49f, (integrin subunit alpha 6, ITGA6), is also identified to be a CSC surface marker, and found to correlate with tumor spheres formation capacity and in vivo selfrenewal ability in lung cancer [31]. In glioblastoma CSCs (GCSCs), CD49f has also been proposed to be an important regulator of stemness [32]. It has been reported that $\mathrm{CD} 49 \mathrm{f}$ is associated with radiation therapy resistance and $\mathrm{CD} 49 \mathrm{f}+$ population-mediated taxane resistance $[33,34]$.

$C D 24$, also known as heat-stable antigen in mouse, is significantly upregulated in different cancers compared to their benign counterparts. In many human cancers, CD24 overexpression is highly associated with adverse prognostic features such as lymph node metastases, advanced clinical stage and shorter overall survival [35]. It is identified to be a CSC marker in bladder cancer [36], hepatocellular carcinoma [37]. In Terence Kin Wah Lee' s study, CD24+ cells were more quiescent, with a greater ability to form tumors in Non-obese diabetic (NOD) /Severe combined immune deficient (SCID) mice, and 
an ability to self-renew, differentiate, and metastasize, especially upregulated in residual resistant tumors upon cisplatin treatment when compared with untreated tumors [37].

$C D 9$, motility-related protein-1, is involved in cell fusion, adhesion, motility, proliferation, metastases and signaling [38, 39]. Some studies have reported that CSCs can contribute towards tumor formation by upregulating $\mathrm{CD}$, thereby maintaining the tumor cell population [40, 41]. In human B-acute lymphoblastic leukemia, CD9 plays important roles in attributing CSC properties, and the CD9+ cells exhibited therapy resistance [42].

\section{Deregulated developmental signaling that govern CSCs and therapy resistance}

Hippo/YAP1 pathway is a highly conserved signaling pathway that regulates cell fate, apoptosis, proliferation and stem cell maintenance in various species [43, 44]. The Hippo pathway components, including a major kinase cascade and scaffold proteins, have been established in both Drosophila and mammals [45]. In mammals, the Hippo pathway consists of a core kinase cascade in which Mst1/2 form a complex with an adaptor protein Sav1 that phosphorylates kinases Lats1/2. Lats1/2 then phosphorylates and represses the transcriptional coactivators YAP1 and TAZ by promoting ubiquitination, degradation, and cytoplasmic retention (Fig. 1a).

Recent emerging data suggest that YAP1 regulate CSCs properties and confer therapy resistance $[46,47]$. Overexpression of YAP1 and its activation (nuclear localization) are associated with poor prognosis in several tumor types, including gastric adenocarcinoma (GAC). We have demonstrated that YAP1 is overexpressed in gastroesophageal cancer and mediates CSC properties through its target SOX9 [48]. Studies from our laboratory also found that YAP1 strongly mediates chemo- and radiation resistance through upregulation EGFR and CDK6 in esophageal cancer respectively [49, 50]. YAP1 has been reported as conferring resistance to cisplatin in human oral squamous cell carcinoma [51]. Others surmised that YAP1 is often the terminal node of many oncogenic pathways [52] and a signaling hub of the tumor microenviroment [53].

In addition, YAP1 was reported to be responsible for target therapy resistance. Lin L et al. reported that YAP1 enhances resistance to RAF and MEK-targeted cancer therapies resistance, revealing that YAP1 and RAF or MEK combined inhibition of synthetic lethality is a promising strategy to enhance treatment response and patient survival [47]; while Lee JE et al. found that YAP1 inhibition restores the sensitivity of EGFR-TKI in lung adenocarcinoma having primary or acquired EGFR-TKI resistance [54].
It has been reported that another hippo downstream effector, TAZ, promotes the self-renewal and tumorseeding potentials of CSCs and confers CSC-like properties on differentiated non-CSC cells in different cancer contexts [55]. Recent evidence suggests that TAZ is also associated with therapy resistance. Zhan $\mathrm{z}$ et al. reported that TAZ, is associated with drug resistance of pancreatic cancer [56], high expression of TAZ in lung adenocarcinoma cells and cell lines is associated with cisplatin resistance. TAZ inhibition restores the sensitivity of cisplatin through AKT/mTOR signaling in lung adenocarcinoma [57].

Wht/ $\beta$-catenin pathway is an ancient and evolutionarily conserved pathway which regulates embryonic development [57]. The canonical $\mathrm{Wnt} / \beta$-catenin signaling cascades are involved in self-renewal of stem cells and proliferation or differentiation of progenitor cells, whereas non-canonical Wnt signaling cascades are involved in maintenance of stem cells, and directional cell movement (Fig. 1b). Both canonical and non-canonical Wnt signaling cascades play key roles in the development and evolution of CSCs [58].

The abnormal activation of Wnt signaling has implicated in many cancers including hepatoblastoma, colorectal cancer, multiple myeloma, and GAC [59]. It has been shown that factors secreted by fibroblasts, such as hepatocyte growth factor (HGF), activate the $\mathrm{Wnt} / \beta$-catenin pathway and CSC expansion in vivo and in vitro. In a colon cancer model, CSCs with high Wnt signaling activity appear to be adjacent to stromal myofibroblasts, which secrete multiple factors to maintain the active Wnt $/ \beta$-catenin pathway, thereby ensuring the stemness characteristics of its neighboring cells [60]. In malignant pleural mesothelioma, Wnt/GSK3 $\beta / \beta$-catenin pathway is found to be upregulated and Wnt-driven autocrine production of IL- 8 and IL-1 $\beta$ contributes to upregulate ABCB5 which is predictive of poor response to chemotherapy [61]. Emons $\mathrm{G}$ et al. report that chemoradiotherapy resistance in colorectal cancer cells is mediated by Wnt/ $\beta$-catenin signaling [62].

Hedgehog pathway (Hh) is critical during embryonic development. It is involved in the patterning of the neural tube, lung, skin, axial skeleton, and gastrointestinal tract [63]. Binding of Hh ligands, relieves the inhibitory effect of their Patched (PTCH) transmembrane receptors on Smoothened (SMO), which is also located in the cell membrane. Subsequently, the signaling cascade initiated by SMO leads to activation and nuclear localization of GLI transcription factors, which drive expression of Hh target genes; most of the target genes are involved in proliferation, survival, and angiogenesis (Fig. 1c) [64].

Hh signaling has been associated with cell fate determination, self-renewal [65] and CSC regulation in many 


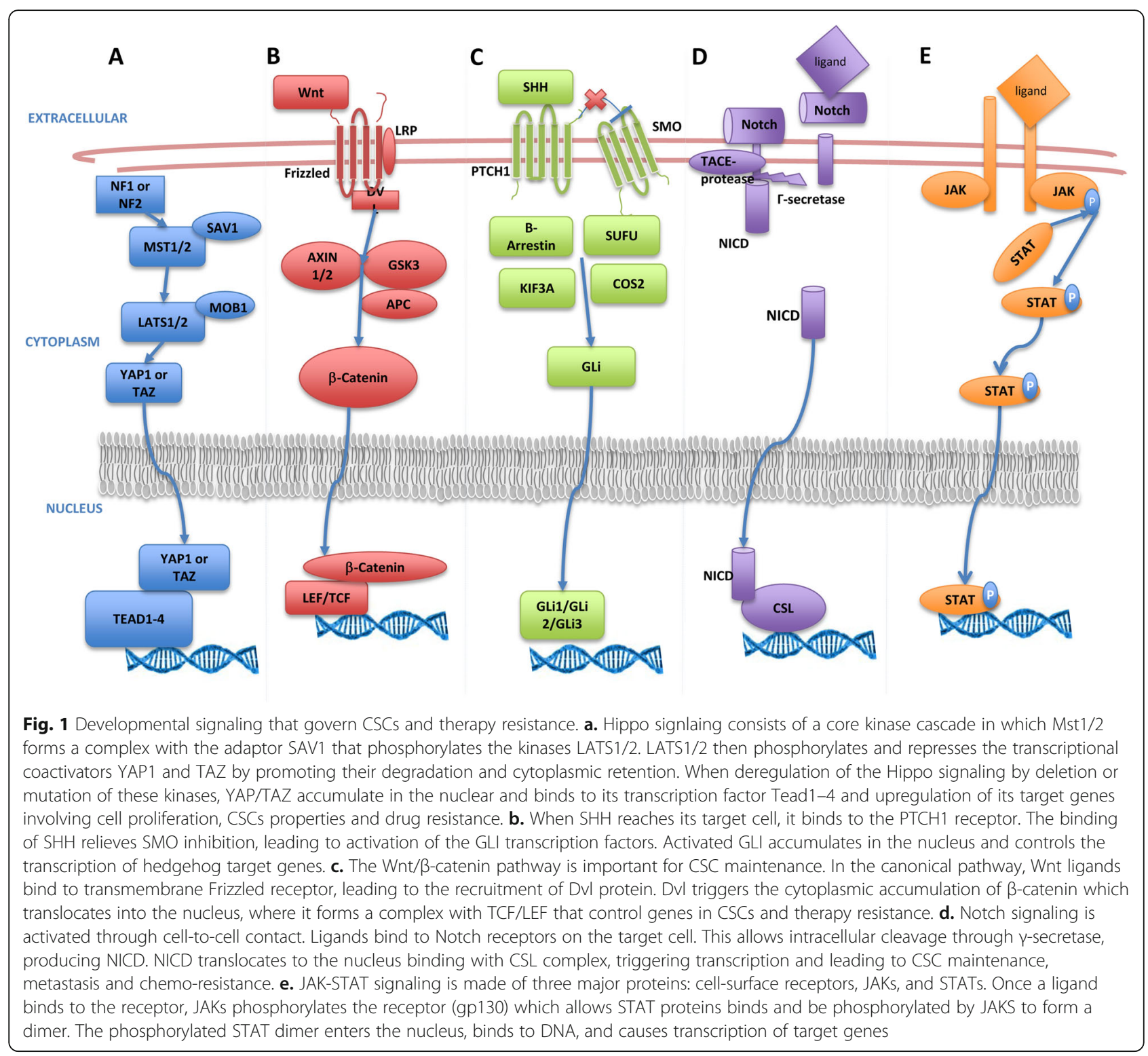

cancer types including leukemias [66], multiple myeloma [67], gliomas [68], breast cancer [69], pancreatic cancer [70], prostate cancer [71], lung cancer [72], melanoma [73], and gastrointestinal cancers [74, 75]. It has been reported that activation of $\mathrm{Hh}$ signaling contributes to therapy resistance and $\mathrm{Hh}$ signaling contributes to tumor regrowth after chemoradiotherapy and a target to improve radiation response [76]. Kobune $M$ found that drug resistance is dramatically restored by Hh inhibitors in CD34+ leukemic cells [77]. Hh signaling affects the sensitivity of hepatoma cells to therapy through the ABCC1 transporter [78].

The Notch pathway, has been extensively explored as a CSC-realted pathways in multiple tumor types. The pathway is activated upon ligand binding to the Notch receptor, which is subsequently cleaved by the ADAM family proteases and $\gamma$-secretase to release the Notch intracellular domain (NICD). NICD translocates to the nucleus, binds to transcription factor CSL, and converts the complex from a repressor to an activator of Notch genes (Fig. 1d). Notch activation has been proposed as vital to CSC populations for maintaining stemness, enhancing therapy resistance, and promoting a hypoxic niche [79]. Notch signaling pathway plays an important role in normal stem cells proliferation, differentiation, and apoptosis. It is also reported that Notch signaling is crucial for cell survival and self-renewal properties of CSCs $[57,80]$.

Notch signaling is one of the most important cascades involved in therapy resistance in tumor cells [71]. Zhang $\mathrm{Y}$ et al. reported that Notch signaling regulates selfrenewal and platinum resistance of CSCs in human non- 
small cell lung cancer [31]. Another report suggested that Notch signaling induces platinum resistance in a HES1-independent manner. Furthermore activation of the Notch signaling pathway is involved in osteosarcoma resistance [81]. Silencing of Notch1 suppressed AKT pathway, reduced EMT, and enhanced the sensitivity of TNBC cells to cisplatin and doxorubicin [82].

JAK/STAT pathway, plays a critical role in various cytokines and growth factors signaling that affect various cellular functions, such as proliferation, growth, and immune response. JAK/STAT signaling is reported to be involved in maintaining embryonic stem cell selfrenewal properties, hematopoiesis, and neurogenesis [83]. It is also reported that inhibiting JAK/STAT pathway can block CSC self-renewal [84]. In the breast cancer, expression of a variety of lipid metabolic genes, including carnitine palmitoyltransferase 1B (CPT1B), which encodes the key enzyme for fatty acid b-oxidation (FAO) is also blocked by the inhibition of JAK/STAT pathway. Human breast-cancer-derived data suggest that the STAT3-CPT1B-FAO pathway promotes cancer cell stemness and therapy resistance. In addition, cells when treated with FDA-approved JAK inhibitor Tofacitinib, became sensitive and underwent apoptosis when combined with doxorubicin [85].

\section{Mechanisms of CSC-mediated therapy resistance}

CSC-mediated therapy resistance appears to be associated with their dormancy/slow-cycling, and/or expression of efflux transporters, avoidance of apoptosis and Non-coding RNAs mediated drug resistance etc.

\section{CSC dormancy, plasticity and drug resistance}

Tumor dormancy, a clinically undetectable state of cancer, contributes the development of multidrug resistance (MDR), minimum residual disease (MRD), tumor outgrowth, cancer relapse, and metastases. CSCs can mediate therapy resistance through dormancy. Cellular dormancy means that cells are recruited into the G0phase of the cell cycle but remain capable of cell division in response to mitotic stimulation [85]. Chemotherapy and irradiation are mainly effective against proliferating cells. It is likely that dormant tumor cells comprise both CSC and non-CSC populations [86]. Study from the Massague J laboratory demonstrated that latency competent cancer (LCC) cells show stem-cell-like characteristics and express SOX2 and SOX9 transcription factors, which are essential for their survival and resistance to therapy in host organs under immune surveillance and for metastatic outgrowth under permissive conditions [87].

\section{ATP-binding cassette transporter ( $A B C$ transporter)}

$A B C$ transporters are proteins that allow transmembrane transportation of different substrates using the energy produced by ATP hydrolysis. These proteins are generally located on the membrane of the cell, which can protect cells from harmful toxins [88]. $A B C$ transporters contain $\mathrm{ABCG} 2, \mathrm{ABCB} 1$, and $\mathrm{ABCC} 1$, etc. Among them, ABCG2 has the ability to transport drugs such as doxorubicin and methotrexate. ABCB1, known as Pglycoprotein, can be expressed in more than half of chemo-resistant tumors [89]. Recent study indicates that downregulating ABCG2 can enhance the chemosensitivity of breast CSCs [90]. Similarly, SUN et al. [91] also found that CSCs showed enhanced chemosensitivity when siRNAs that blocked $\mathrm{ABC}$ transporter expression were added to breast CSC culture media along with drugs. Surprisingly, ABC transporter proteins such as multi-drug resistance protein-1, leading to therapy-resistance of CSCs is controlled by Hh signaling [92]. Therefore, $A B C$ transporter proteins can be used as a surface markers for CSC identification, and their ability to transport drugs itself enables CSCs to prosper.

\section{Avoidance of apoptosis through rho family}

Rho protein, a member of small GTPases, is highly conserved and plays an important role in pathological processes including cancer progression, inflammation and wound repair [93]. Rho-associated protein kinase (ROCK), the effector of Rho, is also proved crucial in cancer progression. Recent study found that the Sox 2 gene can regulate the motility of colorectal cancer cells and promote tumor progression through the Rho-ROCK signaling pathway [94]. In the study of targeting of RhoROCK pathway in melanoma and breast CSCs, CSC motility and invasiveness decreased [95]. Similarly, small molecule inhibitors targeting ROCK could inhibit the expression of survivin by blocking the Rho pathway and increase the sensitivity of pancreatic CSCs to drugs [96]. In conclusion, activation of the Rho-ROCK pathway promotes survivin expression, and survivin acts as an antiapoptotic protein to protect CSCs from therapy-induced apoptosis, thus enabling CSCs to resist therapy and strengthen stemness.

Non-coding RNAs (nc-RNAs), CSCs and therapy resistance Recent studies have shown that different types of ncRNAs, such as microRNAs (miRNAs) and long-chain non-coding RNAs (IncRNAs) can control growth and division of CSCs and disease progression by regulating transcription factors and downstream signaling pathways [97-100] Therefore, the effects of non-coding RNAs on intracellular signaling pathways and cell stemness maintenance are the basis for many diseases including tumors $[100,101]$. Accumulated evidence confirms that miRNAs 
are critical for the maintenance, self-renew, and differentiation of CSCs [102]. For example, study by Liu et al. [103] demonstrated that miR-125b upregulated by the Snail via Wnt pathway can promote CSC hematopoiesis and therapy resistance. In the study of pancreatic cancer, it was reported that simultaneous inhibition of miR-21 and miR-221 can reduce the number of CSCs and reduce differentiation, leading to a decline in the overall proliferative, invasive ability and therapy resistance [104]. While Inc-RNA is a group of nc-RNAs longer than 200 nucleotides, studies have shown that Inc-RNA is closely related to CSCs. Studies in lung adenocarcinoma have shown that IncRNA-ROR regulates the expression of EMT-associated with tumor invasion, metastases, and therapy resistance [105]. Wang et al [106] reported that in prostate cancer, IncRNA HOTAIR can be induced by gemcitabine, and can promote the selfrenewal and migration ability of CSCs. In summary, miRNAs and LncRNAs mainly regulate CSCs by controlling the expression of intracellular proteins and the activation of related signaling pathways, which enables CSCs to maintain their stemness and therapy resistance.

\section{Other stemness genes that mediate therapy resistance Numb protein}

Numb protein, involves cell development, adhesion and migration, cell misalignment, and endotoxin, and ubiquitination of the target protein [107]. In prostate cancer, low or negative Numb CSCs preferentially express Notch and Hh downstream and stem cell-associated genes, enrich a castration-resistant prostate cancer cell subpopulation [107]. Phosphorylation of Numb by NANOG destabilizes Numb-p53 complex, leading to p53 proteolysis, then promotes self-renew and tumorigenesis in liver cancer [108].

\section{Musashi (MSI)}

Musashi, underwent a duplication event in vertebrates giving rise to two homologs: Musashi1 (MSI1) and Musashi2 (MSI2). As a member of RNA binding protein family, it is capable of maintaining the infinite proliferation of stem cells through transcriptional regulation or activation of related protein expression [109]. By downregulating pro-apoptotic genes, overexpression of MSI1 in glioblastoma can protect tumor cells from apoptosis induced by drugs [110]. The study found that MSI1 as a stem gene in colorectal cancer cells is a key regulator of CD $44^{+}$CSC development and enhances tumor stem cell therapy resistance by triggering the formation of antiapoptotic stress granules (SGs) [111]. FANG's study showed that MSI2 can up-regulate the expression level of the self-renewing gene Lin28A in hepatocellular CSCs to achieve CSC therapy resistance, and knock down of MSI2 gene leads to changes in CSC self-renewal and therapy resistance [112]. Therefore, MSI proteins play an important role in the anti-apoptotic process of CSCs that might be the molecular basis for CSCs to resist drugs.

\section{Bmi1}

The chromatin modifier Bmil is required for selfrenewal of hematopoietic stem cells as well as for selfrenewal of neural, mammary-gland, and prostate gland stem cells [113]. Bmi 1 functions by modifying histones and repressing genes that regulate apoptosis (P19 and p53) and senescence (p16) in stem cells but not in their differentiated progeny [114]. It has been reported that Bmil is responsible for the resistance to the tyrosine kinase inhibitors (TKIs) in a $B C R-A B L 1$-independent way and co-expressed CD26+ in leukemic stem cells of chronic myeloid leukemia [115]. Study from Tang D's group demonstrated that Bmil confers resistance to tamoxifen in estrogen receptor positive breast cancer [116].

\section{Toll-like receptors 4 (TLR4)}

TLR4 is a transmembrane protein, its activation leads to initiation of intracellular NF-kB signaling pathway and production of inflammatory cytokines associated with the innate immune system [117]. While NF- $\mathrm{kB}$ plays an important role in the regulation of immune response, and its dysregulation is considered to be related to tumorigenesis. In gliomas, the interaction of lipopolysaccharide (LPS) with TLR4 can induce tumor stem cell proliferation, and therapy resistance. At the same time, the cytotoxic $\mathrm{T}$ cell killing ability can also be alleviated by LPS [118]. In human hepatocellular carcinoma, it reported that the expression of TLR4 was associated with stemness of CSCs and TLR4 promoted tumor invasion, metastases, and might serve as a surface marker for CSCs [119]. Therefore, activation of TLRs and its downstream signal pathways (NF-kB, etc.) enhance the stemness of CSCs and increase the expression level of cytokines (TNF- $\alpha$, IL-6, etc.) that are associated with CSC therapy-resistance.

\section{CSCs niche, TME and drug resistance}

The tumor microenvironment (TME) consists of stromal cells, immune cells, cytokine networks, chemokines and growth factors, hypoxic regions and ECM. TME stimulates CSC self-renewal, angiogenesis and remodeling immunity, providing other environments that are conducive to CSC tumor invasion and metastasis and dynamic changes [120]. CSCs niche modulates the Wnt/ $\beta$-catenin, Notch, and Hh signaling pathways and/or interrupts the master transcriptional regulators like NANOG, OCT-4, and SOX-2 etc. to maintain the stemness of CSCs. Under specific microenvironmental 
stimuli, certain cancer cells exhibit plasticity, which enables them to resume proliferation through EMT [121]. For example, Chang et al [122] revealed that the activation of the Hh signaling pathway in the TME can transform common prostate cells into stem cells. The TME mainly affects the process of tumor progression and evolution, and the CSCs niche that exists in the TME plays an important role in the origin and evolution of the tumor, which proves that the TME has an important influence on the development stages of tumors.

To date, many therapy resistance mechanisms involving the TME and CSCs niche have been identified across cancer types and these mechanisms have been classified into a range of categories including physical barriers to treatment and cell-adhesion-associated drug resistance [123].

\section{Strategies to target CSCs and overcome therapy resistance}

Because of their role in drug resistance and tumor metastasis, CSCs have contributed significantly to adverse outcomes of patients. In terms of reducing CSCs to improve the prognosis of patients, therapies that target key molecules for CSC maintenance seem theoretically feasible. Several new therapies targeting stem-associated genes and pathways have been proposed to specifically eradicating CSCs [124].

\section{Target deregulated CSCs signaling}

As mentioned earlier, dysregulation of developmental signaling pathways has been shown to be associated with the oncogenic function of CSCs. Targeting pathways that lead the normal stem cells to CSCs open a new dimension for treating cancers associated with the high rate of recurrence and therapy resistance. Several novel strategies targeting CSC affecting pathways alone and in combination with different therapeutic agents are in clinical trials. For example, EMT and CSC marker expression were significantly enhanced in resistant ovarian cancer cells, which was accompanied by activation of $\mathrm{PI} 3 \mathrm{~K} / \mathrm{Akt} / \mathrm{mTOR}$ signaling. Compared with single cisplatin treatment, combined treatment with pathway inhibitor and cisplatin significantly disrupted the colony formation ability, induced higher ROS levels and more apoptosis in resistant ovarian cancer cells. Furthermore, the combination approach effectively inhibited the PI3K/ Akt/mTOR signaling pathway, reversed EMT, and decreased CSC marker expression [125]. In liver cancer, the use of curcumin (NF-kB signaling pathway inhibitor) to block NF-kB can specifically target the CSC population, and suggests the potential for the combined inhibition of NF-kB and HDAC signals can be used to treat patients with poor prognosis [126].
In osteosarcoma, tankyrase inhibitor (IWR-1) inhibited tumor progression associated with specific downregulation of TCF/LEF transcriptional activity and nuclear $\beta$-catenin expression, suggesting that targeting the Wnt/ $\beta$-catenin pathway can eliminate the CSC population. In cervical cancer cell line, curcumin inhibited proliferation, invasion, stemness of cervical cancer cells through impairing Wnt $/ \beta$-catenin and NF-K $\beta$ pathways [127] The combination of conventional chemotherapy with $W n t / \beta$-catenin inhibition can improve therapeutic effect by eliminating aggressive osteosarcoma CSCs and reducing therapy resistance [128]. Many Wnt/ $\beta$-catenin signaling inhibitors have been developed and in the preclinical and clinical trials such as PKF115-584 (CGP049090) has been tested and shown inhibiting the growth of HCC cells in xenografts [129].

The importance of Hippo/YAP1 and deregulation of the Hippo pathway during cancer development and progression are emerging [130]. Our recent report demonstrated that, CA3, a novel potent YAP1 small molecule inhibitor effectively suppressed CSC properties and reduced the fraction of ALDH1+ cells enriched in radiation resistant cells [131]. Moreover, CA3 and 5-FU synergistically inhibited EAC growth, especially that of high YAP1-expressing and resistant cells [131]. YAP1 antisense oligo has been tested in the preclinical and clinical trials through collaboration between Ionis and MDACC (unpublished data).

Recently, FDA approved three new drugs that can target CSCs. Vismodegib is a hedgehog inhibitor that targets a subset of CSCs in basal-cell carcinoma [132]. Vismodegib has also been tested in preclinical models and clinical trials in other solid tumors, such as esophageal cancer. The BCL-2 inhibitor venetoclax selectively killed AML stem cells, and demonstrated that $60 \%$ of patients receiving venetoclax (with other agents) had completely clinical response [133]. Similarly, we also found AT101, another pan BCL-2 inhibitor target CSC genesYAP1/SOX9 and proved effective in esophageal and gastric cancer patients in the preclinical and clinical setting (manuscript in GUT, 2021 in press).

\section{Target CSCs markers}

According to characteristics of CSCs, integrin $\alpha v \beta 3$ (integrin $\alpha v \beta 3$ ) acts as a cell surface adhesion molecule that can induce stemness [134]. In breast cancer, lung cancer, and pancreatic cancer, stemness is induced by KRas/ $\mathrm{RalB} / \mathrm{NF}-\mathrm{kB}$, which is expected to be an effective target for inhibiting stemness [135]. In nasopharyngeal carcinoma, FoxM1 is significantly associated with stem cellrelated clinicopathological features, including advanced clinical stages, tumor recurrence, and distant metastases. At the same time, FoxM1 is closely related to the expression levels of stem cell markers such as Nanog, Sox2 
and OCT4 in tumor samples, and promoted the expression of these stem-related genes in vitro. In addition, FoxM1 gives cancer cells self-renewal properties by increasing the side population (SP) cells and forms larger and more numerous tumor spheres [136]. Song et al. [137] found that the mitochondrial membrane of CSCs has a high PRX3 gene expression in colon cancer cells. FoxM1 can stimulate overexpression of CD133 and PRX3, to regulate the stemness of colon cancer stem cells. In addition, targeting glioma stem cells through combined BMI1 and EZH2 inhibition proved more effective than either agent alone both in culture and in vivo, suggesting that strategies that simultaneously target multiple epigenetic regulators within glioblastomas may be effective in overcoming therapy resistance caused by ITH [138].

\section{Targeting miRNA/LncRNAs that associated with CSCs}

Recently, lncRNA and miRNA have been discovered as new targets for affecting stemness. In triple-negative breast cancer (TNBC), LNC00284, also known as LNCR NA-NRAD1, is associated with worth patient outcomes. Targeting NRAD1 in TNBC tumors using antisense oligonucleotides reduced cell survival, tumor growth, and the number of cells with CSC characteristics [139]. In cholangiocarcinoma (CCA) lnc-PKD2-2-3 increased CD44, CD133 and OCT4 expression as well as the $\mathrm{CD} 44+\mathrm{CD} 133+$ cell proportion, raised tumor sphere forming efficiency and enhanced tharapy resistance to 5FU in TFK-1 and Huh-28 cells. In addition, lnc-PKD22-3 was positively correlated with CSC markers in CCA tumor tissues and was markedly upregulated in CCA stem-like cells compared with that in normal CCA cells [140]. Amit K. Srivastava et al. reported that miR-328-3p (miR-328) is significantly upregulated in ovarian CSCs. High expression of miR-328 maintained CSC properties by directly targeting DNA damage binding protein 2 (DDB2), which has been shown previously to inhibit ovarian CSC. Targeting miR-328 could be exploited for the eradication of CSC and aversion of tumor metastasis in ovarian cancer [57].

\section{Conclusion}

The common features of these molecular mechanisms of CSC-mediated therapy resistance are the maintenance of the stemness and dormancy which is the basis for the ability of CSCs to counteract therapy. Other mechanisms include drug efflux mechanism; anti-apoptotic mechanism; DNA damage repair mechanism, and CSC niche, immune evasion by manipulating the TME. By studying the mechanism of resistance, we can explore new targets and improve traditional anti-tumor strategies. The targeted therapy of CSC has greater potential than the traditional therapy to simultaneously eliminate progenitor cells and CSCs, and improve the overall effect of therapy in cancer patients. Current treatment strategies targeting CSCs mainly include specific surface markers and intracellular signal transduction pathways for CSCs, induction of tumor stem cell differentiation, and alteration of TME. The intra/inter tumor heterogeneity and the complexity of the TME make therapy extremely ineffective, therefore, greater understanding of intra/inter tumor heterogeneity and TME is needed for novel therapies to emerge. Future successful eradiation of CSCs and overcome therapy resistance mainly depend on combination therapies that target multiple CSC pathways as well as target proliferating cancer cells.

\section{Abbreviations \\ GAC: Gastric adenocarcinoma; CSCs: Cancer stem cells; EMT: Epithelial- mesenchymal transition; TME: Tumor micro-environment; ALDH1: Aldehyde dehydrogenase1; HA: Hyaluronic acid; ALCAM: Activated leukocyte cell adhesion molecule; PTC: Papillary thyroid carcinoma; HGF: Hepatocyte growth factor; PTCH: Patched transmembrane receptors; NICD: Notch intracellular domain; CPT1B: Carnitine palmitoyltransferase 1B; FAO: Fatty acid b-oxidation; MDR: Multidrug resistance; MRD: Minimum residual disease; ABC transporter: ATP-binding cassette transporter; ROCK: Rho-associated protein kinase; LncRNAs: Long-chain non-coding RNAs; MSI: Musashi; SGs: Stress granules; TLR4: Toll-like receptors 4; TNBC: Triple-negative breast cancer; CCA: Cholangiocarcinoma; DDB2: Damage binding protein 2; ROS: Reactive oxygen species; RNS: Reactive nitrogen species; ITH: Intratumor heterogeneity; NOD/SCID: Non-obese diabetic/Severe combined immune deficient}

\section{Acknowledgements}

We appreciate Sarah Branson, a scientific editor from Scientific Publications, Research Medical Library at The University of Texas MD Anderson Cancer Center, for her excellent editing of this manuscript.

\section{Authors' contributions}

(I) Conception and design: S. Song, Y Li; (II) Manuscript writing: Y Li, S. Song, J Ajani; (III). Revision and Final approval of manuscript: Y Li, S. Song, Z. Wang, J Ajani. All authors read and approved the final manuscript.

\section{Funding}

This work was supported by an MD Anderson Institutional Research Grant (3-0026317 to S. Song); and grants from Department of Defense (CA160433 and CA170906 to S. Song and CA160445 to J Ajani); and the National Institutes of Health (CA129906, CA138671, and CA172741 to J.A. Ajani).

\section{Availability of data and materials}

Not applicable.

\section{Ethics approval and consent to participate}

The authors are accountable for all aspects of the work in ensuring that questions related to the accuracy or integrity of any part of the work are appropriately investigated and resolved.

Consent for publication

All authors agreed and approved for the publication.

\section{Competing interests}

The authors declare that there are no conflicts of interest.

\section{Author details}

'Department of Gastrointestinal Medical Oncology, The University of Texas MD Anderson Cancer Center, 1515 Holcombe Boulevard, Houston, TX 77030-4009, USA. ${ }^{2}$ Department of Surgical Oncology and General Surgery, First Hospital of China Medical University, Shenyang 110001, People's Republic of China. 
Received: 30 April 2020 Accepted: 9 July 2020

\section{Published online: 15 February 2021}

\section{References}

1. van Neerven SM, Tieken M, Vermeulen $L$, et al. Bidirectional interconversion of stem and non-stem cancer cell populations: a reassessment of theoretical models for tumor heterogeneity. Mol Cell Oncol. 2016:3:e1098791.

2. Ding L, Raphael BJ, Chen F, et al. Advances for studying clonal evolution in cancer. Cancer Lett. 2013;340:212-9.

3. Peiris-Pages M, Martinez-Outschoorn UE, Pestell RG, et al. Cancer stem cell metabolism. Breast Cancer Res. 2016:18:55.

4. Abdullah LN, Chow EK. Mechanisms of chemoresistance in cancer stem cells. Clin Transl Med. 2013;2:3.

5. Allegra A, Alonci A, Penna G, et al. The cancer stem cell hypothesis: a guide to potential molecular targets. Cancer Investig. 2014;32:470-95.

6. Zhao Y, Dong Q, Li J, et al. Targeting cancer stem cells and their niche: perspectives for future therapeutic targets and strategies. Semin Cancer Biol. 2018:53:139-55.

7. Steinbichler TB, Dudas J, Skvortsov S, et al. Therapy resistance mediated by cancer stem cells. Semin Cancer Biol. 2018;53:156-67.

8. Clarke MF, Dick JE, Dirks PB, et al. Cancer stem cells--perspectives on current status and future directions: AACR workshop on cancer stem cells. Cancer Res. 2006;66:9339-44.

9. Dirks PB. Brain tumour stem cells: the undercurrents of human brain cancer and their relationship to neural stem cells. Philos Trans R Soc Lond Ser B Biol Sci. 2008:363:139-52.

10. Ajani JA, Song S, Hochster HS, et al. Cancer stem cells: the promise and the potential. Semin Oncol. 2015:42(Suppl 1):S3-17.

11. Collins AT, Maitland NJ. Prostate cancer stem cells. Eur J Cancer. 2006;42: 1213-8.

12. Wu Y, Wu PY. CD133 as a marker for cancer stem cells: progresses and concerns. Stem Cells Dev. 2009;18:1127-34.

13. Klemba A, Purzycka-Olewiecka JK, Wcislo G, et al. Surface markers of cancer stem-like cells of ovarian cancer and their clinical relevance. Contemp Oncol (Pozn). 2018:22:48-55.

14. Attia S, Atwan N, Arafa M, et al. Expression of CD133 as a cancer stem cell marker in invasive gastric carcinoma. Pathologica. 2019;111:18-23.

15. Yiming L, Yunshan G, Bo M, et al. CD133 overexpression correlates with clinicopathological features of gastric cancer patients and its impact on survival: a systematic review and meta-analysis. Oncotarget. 2015;6: 42019-27.

16. Liu YP, Yang CJ, Huang MS, et al. Cisplatin selects for multidrug-resistant CD133+ cells in lung adenocarcinoma by activating notch signaling. Cancer Res. 2013;73:406-16.

17. Lu R, Zhao G, Yang Y, et al. Inhibition of CD133 overcomes Cisplatin resistance through inhibiting PI3K/AKT/mTOR signaling pathway and autophagy in CD133-positive gastric Cancer cells. Technol Cancer Res Treat. 2019;18:1533033819864311.

18. Yan Y, Zuo X, Wei D. Concise review: emerging role of CD44 in Cancer stem cells: a promising biomarker and therapeutic target. Stem Cells Transl Med. 2015;4:1033-43.

19. Wang L, Zuo X, Xie K, et al. The role of CD44 and Cancer stem cells. Methods Mol Biol. 2018;1692:31-42.

20. Pors K, Moreb JS. Aldehyde dehydrogenases in cancer: an opportunity for biomarker and drug development? Drug Discov Today. 2014;19:1953-63.

21. Raha D, Wilson TR, Peng J, et al. The cancer stem cell marker aldehyde dehydrogenase is required to maintain a drug-tolerant tumor cell subpopulation. Cancer Res. 2014;74:3579-90.

22. Ajani JA, Wang X, Song S, et al. ALDH-1 expression levels predict response or resistance to preoperative chemoradiation in resectable esophageal cancer patients. Mol Oncol. 2014;8:142-9.

23. Meng $E_{1}$ Mitra $A$, Tripathi $K$, et al. ALDH1A1 maintains ovarian cancer stem cell-like properties by altered regulation of cell cycle checkpoint and DNA repair network signaling. PLoS One. 2014;9:e107142.

24. Nguyen $\mathrm{PH}$, Giraud J, Chambonnier L, et al. Characterization of biomarkers of tumorigenic and Chemoresistant Cancer stem cells in human gastric carcinoma. Clin Cancer Res. 2017:23:1586-97.

25. Shouval R, Furie N, Raanani P, et al. Autologous hematopoietic stem cell transplantation for systemic sclerosis: a systematic review and meta-analysis. Biol Blood Marrow Transplant. 2018;24:937-44.
26. Donovan MJ, Cordon-Cardo C. Predicting high-risk disease using tissue biomarkers. Curr Opin Urol. 2013:23:245-51.

27. Ofori-Acquah SF, King JA. Activated leukocyte cell adhesion molecule: a new paradox in cancer. Transl Res. 2008;151:122-8.

28. Kim HM, Koo JS. Immunohistochemical analysis of Cancer stem cell marker expression in papillary thyroid Cancer. Front Endocrinol (Lausanne). 2019;10: 523.

29. Xiao M, Yan M, Zhang J, et al. Cancer stem-like cell related protein CD166 degrades through E3 ubiquitin ligase CHIP in head and neck cancer. Exp Cell Res. 2017;353:46-53.

30. Nazilah Abdul Satar KSF, Lim MN, Zakaria PLMN, Fakharuzi NA, Rahman AZA BHYaPB ZZ. Novel triple-positive markers identified in human non-small cell lung cancer cell line with chemotherapy-resistant and putative cancer stem cell characteristics. Oncol Rep. 2018;40:669-81.

31. Zhang $Y, X u$ W, Guo H, et al. NOTCH1 signaling regulates self-renewal and platinum Chemoresistance of Cancer stem-like cells in human non-smal cell lung Cancer. Cancer Res. 2017:77:3082-91.

32. Lathia JD, Gallagher J, Heddleston JM, et al. Integrin alpha 6 regulates glioblastoma stem cells. Cell Stem Cell. 2010;6:421-32.

33. Gomez-Miragaya J, Palafox M, Pare L, et al. Resistance to Taxanes in triplenegative breast Cancer associates with the dynamics of a CD49f+tumorinitiating population. Stem Cell Reports. 2017;8:1392-407.

34. Hu T, Zhou R, Zhao $Y$, et al. Integrin alpha6/Akt/Erk signaling is essential for human breast cancer resistance to radiotherapy. Sci Rep. 2016;6:33376.

35. Lee JH, Kim SH, Lee ES, et al. CD24 overexpression in cancer development and progression: a meta-analysis. Oncol Rep. 2009;22:1149-56.

36. Ooki A, VandenBussche CJ, Kates M, et al. CD24 regulates cancer stem cell (CSC)-like traits and a panel of CSC-related molecules serves as a noninvasive urinary biomarker for the detection of bladder cancer. $\mathrm{Br} \mathrm{J}$ Cancer. 2018;119:961-70.

37. Lee TK, Castilho A, Cheung VC, et al. CD24(+) liver tumor-initiating cells drive self-renewal and tumor initiation through STAT3-mediated NANOG regulation. Cell Stem Cell. 2011;9:50-63.

38. Hemler ME. Tetraspanin proteins mediate cellular penetration, invasion, and fusion events and define a novel type of membrane microdomain. Annu Rev Cell Dev Biol. 2003;19:397-422.

39. Lin Q, Peng S, Yang Y. Inhibition of CD9 expression reduces the metastatic capacity of human hepatocellular carcinoma cell line MHCC97-H. Int J Oncol. 2018;53:266-74

40. Podergajs N, Motaln H, Rajcevic $U$, et al. Transmembrane protein CD9 is glioblastoma biomarker, relevant for maintenance of glioblastoma stem cells. Oncotarget. 2016;7:593-609.

41. Wang VM, Ferreira RMM, Almagro J, et al. CD9 identifies pancreatic cancer stem cells and modulates glutamine metabolism to fuel tumour growth Nat Cell Biol. 2019:21:1425-35.

42. Yamazaki $\mathrm{H}, \mathrm{Xu} \mathrm{CW}$, Naito $\mathrm{M}$, et al. Regulation of cancer stem cell properties by CD9 in human B-acute lymphoblastic leukemia. Biochem Biophys Res Commun. 2011;409:14-21.

43. Harvey KF, Pfleger CM, Hariharan IK. The Drosophila Mst Ortholog, hippo, restricts growth and cell proliferation and promotes apoptosis. Cell. 2003; 114:457-67.

44. Yu FX, Zhao B, Guan KL. Hippo pathway in organ size control, tissue homeostasis, and Cancer. Cell. 2015:163:811-28.

45. Pan D. The hippo signaling pathway in development and cancer. Dev Cell. 2010;19:491-505.

46. Moroishi T, Hansen CG, Guan KL. The emerging roles of YAP and TAZ in cancer. Nat Rev Cancer. 2015;15:73-9.

47. Lin L, Sabnis AJ, Chan E, et al. The hippo effector YAP promotes resistance to RAF- and MEK-targeted cancer therapies. Nat Genet. 2015:47:250-6.

48. Wei W, Chua MS, Grepper S, et al. Blockade of Wnt-1 signaling leads to antitumor effects in hepatocellular carcinoma cells. Mol Cancer. 2009;8:76.

49. Song S, Maru DM, Ajani JA, et al. Loss of TGF-beta adaptor beta2SP activates notch signaling and SOX9 expression in esophageal adenocarcinoma. Cancer Res. 2013:73:2159-69.

50. Li F, Xu Y, Liu B, et al. YAP1-mediated CDK6 activation confers radiation resistance in esophageal Cancer - rationale for the combination of YAP1 and CDK4/6 inhibitors in esophageal Cancer. Clin Cancer Res. 2019:25:2264-77.

51. Yoshikawa K, Noguchi K, Nakano Y, et al. The hippo pathway transcriptional co-activator, YAP, confers resistance to cisplatin in human oral squamous cell carcinoma. Int J Oncol. 2015;46:2364-70. 
52. Keren-Paz A, Emmanuel R, Samuels Y. YAP and the drug resistance highway. Nat Genet. 2015;47:193-4.

53. Zanconato F, Cordenonsi M, Piccolo S. YAP and TAZ: a signalling hub of the tumour microenvironment. Nat Rev Cancer. 2019;19:454-64.

54. Lee JE, Park HS, Lee D, et al. Hippo pathway effector YAP inhibition restores the sensitivity of EGFR-TKI in lung adenocarcinoma having primary or acquired EGFR-TKI resistance. Biochem Biophys Res Commun. 2016:474:154-60

55. Li Z, Wang Y, Zhu Y, et al. The hippo transducer TAZ promotes epithelial to mesenchymal transition and cancer stem cell maintenance in oral cancer. Mol Oncol. 2015;9:1091-105.

56. Zhan T, Huang $X$, Tian $X$, et al. Downregulation of MicroRNA-455-3p links to proliferation and drug resistance of pancreatic Cancer cells via targeting TAZ. Mol Ther Nucleic Acids. 2018;10:215-26.

57. Clevers H, et al. Wnt/beta-catenin signaling and Disease. Cell. 2012.

58. Katoh M. Canonical and non-canonical WNT signaling in cancer stem cells and their niches: cellular heterogeneity, omics reprogramming, targeted therapy and tumor plasticity (review). Int J Oncol. 2017;51:1357-69.

59. Kahn M. Wnt signaling in stem cells and Cancer stem cells: a tale of two Coactivators. Prog Mol Biol Transl Sci. 2018;153:209-44.

60. Vermeulen $L$, De Sousa EMF, van der Heijden M, et al. Wnt activity defines colon cancer stem cells and is regulated by the microenvironment. Nat Cell Biol. 2010;12:468-76.

61. Milosevic V, Kopecka J, Salaroglio IC, et al. Wnt//L-1 beta/LL-8 autocrine circuitries control chemoresistance in mesothelioma initiating cells by inducing ABCB5. Int J Cancer. 2019.

62. Emons $G$, Spitzner M, Reineke $\mathrm{S}$, et al. Chemoradiotherapy resistance in colorectal Cancer cells is mediated by Wnt/beta-catenin signaling. Mol Cancer Res. 2017;15:1481-90.

63. Chiang C, Ying $L T T$, Lee $E$, et al. Cyclopia and defective axial patterning in mice lacking sonic hedgehog gene function. Nature. 1996;383:407-13.

64. Beachy PA, Hymowitz SG, Lazarus RA, et al. Interactions between hedgehog proteins and their binding partners come into view. Genes Dev. 2010;24: 2001-12.

65. Cochrane CR, Szczepny A, Watkins DN, et al. Hedgehog signaling in the maintenance of Cancer stem cells. Cancers (Basel). 2015:7:1554-85.

66. Zhao C, Chen A, Jamieson $\mathrm{CH}$, et al. Hedgehog signalling is essential for maintenance of cancer stem cells in myeloid leukaemia. Nature. 2009:458:776-9.

67. Peacock CD, Wang QJ, Gesell GS, et al. Hedgehog signaling maintains a tumor stem cell compartment in multiple myeloma. Proc Natl Acad Sci U S A. 2007;104:4048-53.

68. Morgenroth A, Vogg AT, Ermert K, et al. Hedgehog signaling sensitizes glioma stem cells to endogenous nano-irradiation. Oncotarget. 2014;5: 5483-93.

69. Kasper M, Jaks V, Fiaschi M, et al. Hedgehog signalling in breast cancer. Carcinogenesis. 2009;30:903-11.

70. Xie J, Rodova M, Fu J, et al. Sonic hedgehog signaling inhibition provides opportunities for targeted therapy by Sulforaphane in regulating pancreatic Cancer stem cell self-renewal. PLoS One. 2012:7.

71. Domingo-Domenech J, Vidal SJ, Rodriguez-Bravo V, et al. Suppression of acquired docetaxel resistance in prostate cancer through depletion of notch- and hedgehog-dependent tumor-initiating cells. Cancer Cell. 2012; 22:373-88.

72. Park KS, Martelotto LG, Peifer M, et al. A crucial requirement for hedgehog signaling in small cell lung cancer. Nat Med. 2011;17:1504-8.

73. Santini R, Vinci MC, Pandolfi S, et al. Hedgehog-GLI signaling drives selfrenewal and tumorigenicity of human melanoma-initiating cells. Stem Cells. 2012;30:1808-18.

74. Yoon C, Park DJ, Schmidt B, et al. CD44 expression denotes a subpopulation of gastric cancer cells in which hedgehog signaling promotes chemotherapy resistance. Clin Cancer Res. 2014:20:3974-88.

75. Varnat F, Duquet A, Malerba M, et al. Human colon cancer epithelial cells harbour active HEDGEHOG-GLI signalling that is essential for tumour growth, recurrence, metastasis and stem cell survival and expansion. EMBO Mol Med. 2009;1:338-51.

76. Sims-Mourtada J, Izzo JG, Apisarnthanarax S, et al. Hedgehog: an attribute to tumor regrowth after chemoradiotherapy and a target to improve radiation response. Clin Cancer Res. 2006;12:6565-72.

77. Kobune $\mathrm{M}$, Takimoto $\mathrm{R}$, Murase $\mathrm{K}$, et al. Drug resistance is dramatically restored by hedgehog inhibitors in CD34+ leukemic cells. Cancer Sci. 2009; 100:948-55.
78. Ding J, Zhou XT, Zou HY, et al. Hedgehog signaling pathway affects the sensitivity of hepatoma cells to drug therapy through the ABCC1 transporter. Lab Investig. 2017;97:819-32.

79. Desai A, Yan Y, Gerson SL. Concise reviews: Cancer stem cell targeted therapies: toward clinical success. Stem Cells Transl Med. 2019;8:75-81.

80. Takebe N, Miele L, Harris PJ, et al. Targeting notch, hedgehog, and Wnt pathways in cancer stem cells: clinical update. Nat Rev Clin Oncol. 2015;12: 445-64.

81. Dai G, Deng S, Guo W. Notch pathway inhibition using DAPT, a gammasecretase inhibitor (GSI), enhances the antitumor effect of cisplatin in resistant osteosarcoma. 2019;58:3-18.

82. Xiao YS, Zeng LYK, et al. Major vault protein is a direct target of Notch1 signaling and contributes to chemoresistance in triple-negative breast cancer cells. Cancer Lett. 2019;440-441:156-67.

83. Matsui WH. Cancer stem cell signaling pathways. Medicine (Baltimore). 2016; 95:S8-s19.

84. Wang T, Fahrmann JF, Lee H, et al. JAK/STAT3-Regulated Fatty Acid betaOxidation Is Critical for Breast Cancer Stem Cell Self-Renewal and Chemoresistance. Cell Metab. 2018:27:136-50 e5.

85. Kleffel S, Schatton T. Tumor dormancy and cancer stem cells: two sides of the same coin? Adv Exp Med Biol. 2013:734:145-79.

86. Nascimento AS, et al. Phosphoproteome profiling reveals critical role of JAKSTAT signaling in maintaining chemoresistance in breast cancer. Oncotarget. 2017

87. Malladi S, Macalinao DG, Jin X, et al. Metastatic latency and immune evasion through Autocrine inhibition of WNT. Cell. 2016;165:45-60.

88. Dai D, Wang $H$, Zhu L, et al. N6-methyladenosine links RNA metabolism to cancer progression. Cell Death Dis. 2018:9:124.

89. Moitra K. Overcoming multidrug resistance in Cancer stem cells. Biomed Res Int. 2015;2015:635745.

90. Das $S$, Mukherjee $P$, Chatterjee $R$, et al. Enhancing Chemosensitivity of breast Cancer stem cells by Downregulating SOX2 and ABCG2 using Wedelolactoneencapsulated nanoparticles. Mol Cancer Ther. 2019;18:680-92.

91. Sun M, Yang C, Zheng J, et al. Enhanced efficacy of chemotherapy for breast cancer stem cells by simultaneous suppression of multidrug resistance and antiapoptotic cellular defense. Acta Biomater. 2015;28:171-82.

92. Sari IN, Phi LTH, Jun N, et al. Hedgehog signaling in Cancer: a prospective therapeutic target for eradicating Cancer stem cells. Cells. 2018;7.

93. Haga RB, Ridley AJ. Rho GTPases: regulation and roles in cancer cell biology. Small GTPases. 2016;7:207-21.

94. Yousefnia S, Momenzadeh S, Seyed Forootan F, et al. The influence of peroxisome proliferator-activated receptor gamma (PPARgamma) ligands on cancer cell tumorigenicity. Gene. 2018;649:14-22.

95. Yang B, Papoian T. Preclinical approaches to assess potential kinase inhibitor-induced cardiac toxicity: past, present and future. J Appl Toxicol. 2018;38:790-800.

96. Takeda H, Okada M, Suzuki S, et al. Rho-associated protein kinase (ROCK) inhibitors inhibit Survivin expression and sensitize pancreatic Cancer stem cells to gemcitabine. Anticancer Res. 2016:36:6311-8.

97. Nahand JS, Taghizadeh-Boroujeni S, Karimzadeh M, et al. microRNAs: new prognostic, diagnostic, and therapeutic biomarkers in cervical cancer. J Cell Physiol. 2019;234:17064-99.

98. Naeli P, Pourhanifeh MH, Karimzadeh MR, et al. Circular RNAs and gastrointestinal cancers: epigenetic regulators with a prognostic and therapeutic role. Crit Rev Oncol Hematol. 2020;145:102854.

99. Yan H, Bu P. Non-coding RNAs in cancer stem cells. Cancer Lett. 2018;421: 121-6.

100. Davis BN, Hata A. Regulation of MicroRNA biogenesis: a miRiad of mechanisms. Cell Commun Signal. 2009;7:18.

101. Shabaninejad Z, Vafadar A, Movahedpour A, et al. Circular RNAs in cancer: new insights into functions and implications in ovarian cancer. J Ovarian Res. 2019:12:84

102. Chhabra R, Saini N. MicroRNAs in cancer stem cells: current status and future directions. Tumour Biol. 2014;35:8395-405.

103. Liu Z, Liu H, Desai S, et al. miR-125b functions as a key mediator for snailinduced stem cell propagation and chemoresistance. J Biol Chem. 2013;288: 4334-45.

104. Zhao Y, Zhao L, Ischenko I, et al. Antisense inhibition of microRNA-21 and microRNA-221 in tumor-initiating stem-like cells modulates tumorigenesis, metastasis, and chemotherapy resistance in pancreatic cancer. Target Oncol. 2015;10:535-48. 
105. Smith M, Zakrzewski J, James S, et al. Posttransplant chimeric antigen receptor therapy. Blood. 2018;131:1045-52.

106. Wang L, Dong P, Wang W, et al. Gemcitabine treatment causes resistance and malignancy of pancreatic cancer stem-like cells via induction of IncRNA HOTAIR. Exp Ther Med. 2017;14:4773-80.

107. Guo Y, Zhang $K$, Cheng $C$, et al. Numb(-/low) enriches a castration-resistant prostate Cancer cell subpopulation associated with enhanced notch and hedgehog signaling. Clin Cancer Res. 2017;23:6744-56.

108. Siddique HR, Feldman DE, Chen $C L$, et al. NUMB phosphorylation destabilizes p53 and promotes self-renewal of tumor-initiating cells by a NANOG-dependent mechanism in liver cancer. Hepatology. 2015;62: $1466-79$.

109. Gulino A, et al. The multiple funcitons of Numb. Exp Cell Res. 2010.

110. Galluzzi L, Vitale I, Aaronson SA, et al. Molecular mechanisms of cell death: recommendations of the nomenclature committee on cell death 2018. Cell Death Differ. 2018;25:486-541.

111. Chiou GY, Yang TW, Huang CC, et al. Musashi-1 promotes a cancer stem cell lineage and chemoresistance in colorectal cancer cells. Sci Rep. 2017;7: 2172.

112. Fang $T$, Lv H, Wu F, et al. Musashi 2 contributes to the stemness and chemoresistance of liver cancer stem cells via LIN28A activation. Cancer Lett. 2017;384:50-9.

113. Lukacs RU, Memarzadeh $\mathrm{S}, \mathrm{Wu} \mathrm{H}$, et al. Bmi-1 is a crucial regulator of prostate stem cell self-renewal and malignant transformation. Cell Stem Cell. 2010;7:682-93.

114. Park IK, Qian DL, Kiel M, et al. Bmi-1 is required for maintenance of adult self-renewing haematopoietic stem cells. Nature. 2003;423:302-5.

115. Galimberti S, Grassi S, Barate C, et al. The Polycomb BMl1 protein is coexpressed with CD26+ in leukemic stem cells of chronic myeloid leukemia. Front Oncol. 2018:8:555.

116. Ojo D, Lin X, Wu Y, et al. Polycomb complex protein BMI1 confers resistance to tamoxifen in estrogen receptor positive breast cancer. Cancer Lett. 2018; 426:4-13.

117. Vaure C, Liu Y. A comparative review of toll-like receptor 4 expression and functionality in different animal species. Front Immunol. 2014;5:316.

118. Steiner RE, Romaguera J, Wang M. Current trials for frontline therapy of mantle cell lymphoma. J Hematol Oncol. 2018;11:13.

119. Liu W-T, Jing Y-Y, Yu G-f, et al. Toll like receptor 4 facilitates invasion and migration as a cancer stem cell marker in hepatocellular carcinoma. Cancer Lett. 2015;358:136-43.

120. Oskarsson T, Batlle E, Massague J. Metastatic stem cells: sources, niches, and vital pathways. Cell Stem Cell. 2014;14:306-21.

121. Gupta R, Bhatt LK, Johnston TP, et al. Colon cancer stem cells: potential target for the treatment of colorectal cancer. Cancer Biol Ther. 2019;20: 1068-82.

122. Chang $\mathrm{HH}$, Chen BY, Wu CY, et al. Hedgehog overexpression leads to the formation of prostate cancer stem cells with metastatic property irrespective of androgen receptor expression in the mouse model. J Biomed Sci. 2011;18:6.

123. Brown Y, Hua S, Tanwar PS. Extracellular matrix-mediated regulation of Cancer stem cells and Chemoresistance. Int J Biochem Cell Biol. 2019.

124. Fu Y, Du P, Zhao J, et al. Gastric Cancer stem cells: mechanisms and therapeutic approaches. Yonsei Med J. 2018:59:1150-8.

125. Deng J, Bai $X$, Feng $X$, et al. Inhibition of PI3K/Akt/mTOR signaling pathway alleviates ovarian cancer chemoresistance through reversing epithelialmesenchymal transition and decreasing cancer stem cell marker expression. BMC Cancer. 2019;19:618.

126. Marquardt JU, Gomez-Quiroz L, Arreguin Camacho LO, et al. Curcumin effectively inhibits oncogenic NF-kappaB signaling and restrains stemness features in liver cancer. J Hepatol. 2015;63:661-9.

127. Ghasemi F, Shafiee M, Banikazemi Z, et al. Curcumin inhibits NF-kB and Wnt/beta-catenin pathways in cervical cancer cells. Pathol Res Pract. 2019; 215:152556.

128. Martins-Neves SR, Paiva-Oliveira DI, Fontes-Ribeiro C, et al. IWR-1, a tankyrase inhibitor, attenuates Wnt/beta-catenin signaling in cancer stemlike cells and inhibits in vivo the growth of a subcutaneous human osteosarcoma xenograft. Cancer Lett. 2018:414:1-15.

129. Song S, Ajani JA, Honjo S, et al. Hippo coactivator YAP1 upregulates SOX9 and endows esophageal cancer cells with stem-like properties. Cancer Res. 2014:74:4170-82.
130. Song S, Xie M, Scott AW, et al. A novel YAP1 inhibitor targets CSC-enriched radiation-resistant cells and exerts strong antitumor activity in esophageal adenocarcinoma. Mol Cancer Ther. 2018;17:443-54.

131. Sekulic A, Migden MR, Lewis K, et al. Pivotal ERIVANCE basal cell carcinoma (BCC) study: 12-month update of efficacy and safety of vismodegib in advanced BCC. J Am Acad Dermatol. 2015;72:1021-6 e8.

132. Pollyea DA, Stevens BM, Jones $C L$, et al. Venetoclax with azacitidine disrupts energy metabolism and targets leukemia stem cells in patients with acute myeloid leukemia. Nat Med. 2018;24:1859-66.

133. Cao J, Li J, Sun L, et al. Hypoxia-driven paracrine osteopontin/integrin alphavbeta3 signaling promotes pancreatic cancer cell epithelialmesenchymal transition and cancer stem cell-like properties by modulating forkhead box protein M1. Mol Oncol. 2019;13:228-45.

134. Seguin L, Kato S, Franovic A, et al. An integrin beta (3)-KRAS-RalB complex drives tumour stemness and resistance to EGFR inhibition. Nat Cell Biol. 2014;16:457-68

135. Luo W, Gao F, Li S, et al. FoxM1 promotes cell proliferation, invasion, and stem cell properties in nasopharyngeal carcinoma. Front Oncol. 2018;8:483.

136. Song IS, Jeong YJ, Jeong SH, et al. FOXM1-Induced PRX3 Regulates Stemness and Survival of Colon Cancer Cells via Maintenance of Mitochondrial Function. Gastroenterology. 2015;149:1006-16 e9.

137. Jin $X$, Kim $L Y$, Wu Q, et al. Targeting glioma stem cells through combined BMI1 and EZH2 inhibition. Nat Med. 2017;23:1352-61.

138. Vidovic D, Huynh $\Pi$, Konda $P$, et al. ALDH1A3-regulated long non-coding RNA NRAD1 is a potential novel target for triple-negative breast tumors and cancer stem cells. Cell Death Differ. 2019.

139. Hourigan CS, Qiu G, Ma D, et al. Lnc-PKD2-2-3, identified by long noncoding RNA expression profiling, is associated with pejorative tumor features and poor prognosis, enhances cancer stemness and may serve as cancer stem-cell marker in cholangiocarcinoma. Proc Natl Acad Sci U S A. 2019;55:45-58.

140. Srivastava AK, Banerjee A. Inhibition of miR-328-3p impairs Cancer stem cell function and prevents metastasis in ovarian. Cancer. 2019;79:2314-26.

\section{Publisher's Note}

Springer Nature remains neutral with regard to jurisdictional claims in published maps and institutional affiliations.

Ready to submit your research? Choose BMC and benefit from:

- fast, convenient online submission

- thorough peer review by experienced researchers in your field

- rapid publication on acceptance

- support for research data, including large and complex data types

- gold Open Access which fosters wider collaboration and increased citations

- maximum visibility for your research: over $100 \mathrm{M}$ website views per year

At $\mathrm{BMC}$, research is always in progress.

Learn more biomedcentral.com/submission 